\title{
Effects of Age and Syntactic Complexity on Speech Motor Performance
}

Christopher Dromey

Brigham Young University, dromey@byu.edu

Kelsey Boyce

Brigham Young University

Ron Channell

Brigham Young University

Follow this and additional works at: https://scholarsarchive.byu.edu/facpub

Part of the Communication Sciences and Disorders Commons

\section{Original Publication Citation}

Dromey, C., Boyce, K., \& Channell, R.W. (2014). Effects of age and syntactic complexity on speech motor performance. Journal of Speech, Language, and Hearing Research, 57, 2142-2151.

\section{BYU ScholarsArchive Citation}

Dromey, Christopher; Boyce, Kelsey; and Channell, Ron, "Effects of Age and Syntactic Complexity on Speech Motor Performance" (2014). Faculty Publications. 1788.

https://scholarsarchive.byu.edu/facpub/1788

This Peer-Reviewed Article is brought to you for free and open access by BYU ScholarsArchive. It has been accepted for inclusion in Faculty Publications by an authorized administrator of BYU ScholarsArchive. For more information, please contact ellen_amatangelo@byu.edu. 
Effects of Age and Syntactic Complexity on Speech Motor Performance

Christopher Dromey, $\mathrm{PhD}$

Kelsey Boyce, MS

Ron Channell, $\mathrm{PhD}$

Department of Communication Disorders

Brigham Young University

Contact Author

Christopher Dromey

133 John Taylor Building

Brigham Young University

Provo, UT 84602

$801-422-6461$ (voice)

801-422-0197 (fax)

dromey@byu.edu 


\begin{abstract}
Purpose: This study investigated the effect of age on articulatory movement and stability in young, middle-aged, and older adults. It also examined the potential influence of linguistic complexity on speech motor control across utterances that differed in their length and grammatical complexity.
\end{abstract}

Method: There were 60 participants in three age groups: 20-30 years, 40-50 years, and 60-70 years, with equal numbers of men and women in each group. The speakers produced 10 repetitions of five different stimuli, each of which included the same bilabial-loaded phrase in different grammatical contexts, while their lip movements were recorded.

Results: Participants from the 60 year-old group had significantly longer utterance durations, while those from the 20 year-old group had the highest jaw spatiotemporal index (STI) values. There were significant differences in the upper lip STI, displacement, and velocity, as well as in vocal intensity for the longer, complex conditions compared to the shorter, phrase-only task. Overall, the differences in performance were minimal across grammatical complexity levels that were equal in length.

Conclusion: These findings suggest that speech motor control matures beyond young adulthood and that linguistic complexity in a repetitive task does not appear to have a consistent effect on measures of speech movement.

Keywords: age; syntactic complexity; articulatory kinematics; speech; language 


\section{Introduction}

During everyday communication both language formulation and speech motor planning require neural resources from the speaker. It could be speculated that since these resources are finite, raising the linguistic demands might lead to compromises in speech movement control. Several studies have addressed this issue in children and in young adults who stutter or who are typically fluent (Maner, Smith, \& Grayson, 2000; Kleinow \& Smith, 2000; Kleinow \& Smith, 2006). However, questions remain about whether older adults show differences in their speech movements as the linguistic difficulty of their utterances is manipulated. Knowing more about the speech performance of typical older adults has the potential to inform our work with disordered speakers by clarifying which changes may be attributed to the aging process itself, as opposed to reflecting a communication disorder.

\section{Developmental Links between Speech and Language}

In discussing the development of spoken language abilities in children, Strand (1992) suggested that many models do not consider the interaction between language and speech production processes, but instead treat them as relatively independent and serial activities. She suggested that understanding the demands of both language and speech on neural processing resources may have clinical implications. For example, it may be helpful to reduce languageprocessing demands when targeting speech accuracy, or reduce motor speech demands when language is the focus of intervention.

More than three decades ago Panagos, Quine, and Klich (1979) reported that children with disordered articulation had a higher count of substitution or omission errors in consonant articulation when they were repeating more complex sentences, such as passive forms. Error rates were also higher when the phonological complexity was greater (disyllables rather than 
monosyllables). Likewise, Abbeduto (1985) reported that syllable durations increased and became more variable during the rapid repetition of complex grammatical forms. In a recent report MacPherson and Smith (2013) noted that numerous studies over the years have shown that disfluencies increase in both children and adults who stutter when they produce longer and/or more complex utterances. Although the children who stuttered in their 2013 study did not show increased kinematic variability for more complex utterances, many had to be excluded from measurement because they were unable to complete the task fluently. This may have masked relevant changes in movement control that might occur as a function of language complexity.

The findings of language and speech interactions in children are of both theoretical and clinical significance. What is not yet clear, however, is how advancing age may influence these interactions. Illes and colleagues (Illes, Metter, Hanson, \& Iritani, 1988) reported decreased syntactic complexity in individuals with advanced Parkinson disease, along with reduced intensity and higher fundamental frequency. The authors speculated that simplifying language might reflect an adaptation on the part of the speakers to increasing dysarthria severity. An investigation of interactions between language structure and speech movements in healthy older adults may help increase our understanding of typical processes in order to distinguish them from the effects of a disorder.

\section{Age Effects on Speech Motor Control}

Previous investigators have compared speech kinematic measures of children with adults (Green, Moore, \& Reilly, 2002; Kleinow et al., 2006; Sharkey \& Folkins, 1985; Murdoch, Cheng, \& Goozee, 2012). Each of these studies has reported higher speech variability in children than adults. Sharkey and Folkins noted that since children's speaking rate is slower, the lower rate may account for the greater variability. This would be consistent with the work of Adams 
and colleagues (Adams, Weismer, \& Kent, 1993), who reported less smooth articulatory gestures at deliberately slow speaking rates. However, it is unclear whether the typically slower speech rate of elderly speakers might influence movement stability in a different way than a volitional reduction in rate. It is possible that speaking at an unaccustomed rate may contribute more to speech movement variability than a slower habitual speaking rate.

Relatively few studies have addressed changes in the speech motor performance of healthy individuals beyond young adulthood. The adults in the Green et al. study ranged in age from 27 to 35 years, and the mean age of the adult speakers in the Kleinow and Smith study was 22 years. Murdoch and colleagues (Murdoch et al., 2012) reported that there was a steady decrease in variability of tongue and lip movement from mid-childhood to adolescence to young adulthood. However, it is possible that the control of articulator movement continues to change with age. One study reported that the accuracy of articulator movement amplitude during a nonspeech visuomotor tracking task increased from childhood to young adulthood, but then declined again with advancing age (Ballard, Robin, Woodworth, \& Zimba, 2001). A recent study (Sadagopan \& Smith, 2013) reported that older adults spoke more slowly, but had similarly stable lip aperture measures to younger adults in a non-word speech repetition task. Another reported that older adults were capable of achieving high peak velocities in jaw movements, but that their fine force control was less precise than in younger speakers (Mefferd \& Corder, 2014). The incidence of communication disorders increases with advancing age. Therefore, in order to better differentiate age- from disorder-related changes in spoken language, it is important to understand how the aging process in healthy individuals impacts speech production as language demands are manipulated. In planning treatment, speech-language pathologists may need to 
consider speech and language interactions in their choice of stimuli, especially if the individuals they treat have deficits in both language and speech.

\section{Utterance Length and Complexity}

Maner, Smith, and Grayson (2000) reported changes in speech motor performance in children and young adults as a function of sentence complexity and length. Their participants spoke an utterance in isolation, followed by that same utterance embedded in longer sentences of varying linguistic complexity. There was higher lip movement variability for the target phrase when it was part of longer, more grammatically complex sentences, as reflected by the spatiotemporal index (STI), a measure of movement steadiness across multiple repetitions of an utterance (Smith, Goffman, Zelaznik, Ying, \& McGillem, 1995). The authors attributed the change in STI to increased utterance length, increased linguistic complexity, or a combination of these factors. No significant differences were noted in STI values between the different levels of complexity (low and high) in the longer utterances. It could be speculated that the complexity conditions used in the Maner et al. study did not provide a great enough range of syntactic forms to cause changes in speech motor performance when sentence length was held constant.

Kleinow and Smith (2000) found that people who stuttered became less consistent in their speech movements for sentences of equal length as linguistic complexity increased, as shown by higher STI values. However, the lip movement stability of the typically fluent control speakers was not affected by grammatical complexity. The authors speculated that their speakers who stuttered, who already had less consistent speech movements than the controls, may have been more susceptible to the added demands of more complex language. What is not clear is whether older speakers may also be more likely than young adults to be influenced by grammatical complexity in their speech motor control. Older adults have been reported to 
perform less well in divided attention studies (Fraser \& Bherer, 2013), where finite neural resources are shared across multiple simultaneous tasks. Although concurrent language formulation and speech movement control cannot be considered divided attention tasks, it could be speculated that increasing linguistic demands may require greater processing resources, and lead to diminished stability in speech movement control in elderly speakers.

\section{Study Aims}

The current study was undertaken to systematically evaluate differences in several kinematic measures of lip and jaw activity during speech as grammatical complexity was manipulated separately from utterance length in three age-groups of speakers. Based on earlier findings of increased lip movement variability in speakers who concurrently completed linguistically or cognitively challenging tasks (Dromey \& Benson, 2003; Dromey \& Bates, 2005), it was hypothesized that producing more complex utterances may tax the available resources and result in higher movement variability. On the basis of studies that have suggested decreasing motor control abilities in older adults (Mefferd et al., 2014; Benjamin, 1997; Harnsberger, Shrivastav, Brown, Jr., Rothman, \& Hollien, 2008), as well as poorer multitasking performance (Fraser et al., 2013), it was hypothesized that producing more grammatically advanced utterances would result in relatively greater kinematic changes in older adults in comparison to younger speakers.

\section{Method}

\section{Participants}

Sixty native English speakers participated in this study. They belonged to one of three age groups: 20 to 30 years $(M=22.95, S D=2.35), 40$ to 50 years $(M=45.25, S D=3.67)$, or 60 
to 70 years $(M=63.20, S D=3.55)$. There were 10 men and 10 women in each age group. The three age groupings were chosen to allow non-overlapping categories reflecting young adulthood, middle age, and older adulthood. It was reasoned that these groups would allow a cross-sectional evaluation of the effects of aging on speech performance under the experimental conditions of varying sentence length and complexity. None of the participants reported any history of speech or language disorders beyond childhood, and all participants communicated comfortably without hearing amplification. Two experimenters were present for each recording, and both verified perceptually normal speaking abilities in all participants. Formal hearing testing was not conducted. Each participant signed an IRB-approved consent document to participate in the study.

\section{Instrumentation}

The speakers' lip and jaw movements were recorded using a head-mounted strain gauge system (Barlow, Cole, \& Abbs, 1983). This device consisted of a lightweight aluminum headset with three cantilever beam strain gauges connected to bridge amplifiers (Biocommunication Electronics model 215) which produced analog voltage signals to record vertical movement of the articulators. To allow a conversion of the voltage signal to a displacement in $\mathrm{mm}$, the cantilever beams were displaced incrementally with a sliding micrometer, and all softwarederived distance and velocity measurements were computed on the basis of this calibration. To measure vertical lip movement, the strain gauge levers were connected to the midline of the upper and lower lip, at the vermillion border. A third lever was attached below the chin to measure vertical jaw movement. Speech was recorded with a microphone (AKG C420) mounted to the headset, and a sound level meter (Extech 407736, C-weighting, fast response) placed 100 $\mathrm{cm}$ from the mouth measured speech intensity in $\mathrm{dB}$ SPL. The signals from these transducers 
were digitized with a Windaq 720 (DATAQ Instruments, Akron, OH) analog/digital converter, with a sampling rate of $1 \mathrm{kHz}$ for the kinematic and sound level meter channels, and $25 \mathrm{kHz}$ (after $12 \mathrm{kHz}$ low pass filtering - Frequency Devices 9002) for the audio channel.

\section{Experimental Procedures}

Sentence stimuli. The sentence stimuli were designed to provide a range of syntactic complexity levels. Cheung and Kemper (1992) compared 11 complexity metrics to evaluate their utility in explaining age-group and individual differences in linguistic complexity. These authors found that mean length of utterance (MLU), the amount of embedding, and the type of embedding were the key factors affecting sentence complexity. Conjoined structures are regarded as less complex and embedded structures as more complex (Givon, 1991).

The five sentences used in the experiment were selected to allow the potential effect of utterance length to be separated from the influence of grammatical complexity for sentences of equal length. These sentences are reported in Table 1. The shortest and simplest utterance was the target phrase open boxes of pompoms in isolation, hereafter referred to as phrase-only. A sentence that included this target phrase was constructed in such a way as to be grammatically simple, but equal in length to the more challenging sentences. In this second condition, the participants read a count of numbers from 1 to 8 , followed by the target phrase, and ending with the same 1 to 8 count. This baseline-counting condition was used as a comparator for the more complex grammatical forms, all of which included the phrase open boxes of pompoms to allow the same kinematic measures to be extracted for each condition. Syntactic complexity of the three remaining sentences was based on the type of embedding and included one conjoined sentence, one sentence containing a single embedded clause (embedding 1), and one sentence containing two embedded clauses (embedding 2). The sentences were equivalent with respect to 
length, each sentence consisting of 17 words, 25 syllables, and an MLU count of 23. The baseline-counting sentence had the same number of syllables as the more grammatically challenging sentences.

The sentence stimuli were presented as 44-point black text on a white background in Microsoft PowerPoint. Participants read the sentences aloud from a 24" computer monitor that was approximately 4 feet from where they were seated. Some of the participants wore eyeglasses but it is not known whether any wore contact lenses. Prior to data collection, the experimenters asked each participant to confirm that they were comfortable reading the large font text. None reported any difficulty reading the sentences.

For the shortest phrase-only stimulus the text remained on the screen for 3 seconds and this was then followed by a blank, white screen for 2 seconds until the next text slide appeared. For the longest sentences the text remained on the screen for 6 seconds with a 2 second blank, white screen between each text presentation. This experimental arrangement allowed each utterance to be produced on a separate breath.

Task performance. All data collection took place within a one-hour session that also involved several tasks for a different experiment. The participants read aloud each sentence when it appeared on the screen (the same sentence 14 times in a block), at a comfortable speaking rate and loudness. The first 10 repetitions were used for analysis, unless one or more included perceptible errors, such as disfluencies, misarticulations, word omissions, or substitutions. Before segmenting the kinematic record, an experimenter (the second author) confirmed the accuracy of each token by listening to the audio recordings and marking for exclusion any utterances with errors. In such cases, one of the four additional tokens was used so that 10 perceptually error-free utterances were included in the analysis. With the exception of one 
participant, who failed to produce 10 acceptable repetitions during the baseline-counting condition, the number of errors within the first 10 tokens ranged from 0 to 3 , with a mean of 0.84. The data for this speaker and condition have been excluded from analysis. The order of utterance block complexity was randomized in order to minimize sequencing effects. The tasks were explained and practiced 5 times before data were collected.

\section{Data Measurement}

The recordings of the upper and lower lip movement were analyzed with custom MATLAB (The Mathworks, 2009) routines. Subsequent mention of the lower lip signal will refer to the combined movements of the lower lip and the jaw (i.e. the jaw contribution to the lower lip movement was not subtracted out). Dependent measures from the entire target phrase included its duration, mean sound pressure level (SPL), and an index of movement variability across repetitions (STI). For one closing bilabial gesture, measures were made of the lower lip displacement and velocity, as well as a correlation between the displacement records for the upper lip and lower lips.

Duration. Utterance duration (ms) was measured from the target phrase, open boxes of pompoms. The measured segment started at the upward velocity peak for the lower lip during the closing bilabial movement of the word open and ended at the upward velocity peak during the closing bilabial movement of the final $/ \mathrm{m} /$ of pompoms (illustrated in Figure 1). The duration was measured to detect possible changes in speaking rate as a function of grammatical complexity.

Displacement and velocity. Displacement of the lower lip was measured for the bilabial closure from $/ \mathrm{a} /$ to $/ \mathrm{m} /$ during the second syllable of the word pompom from each token. The peak velocity from this closing movement was also computed. These measures were made to 
better understand how the grammatical complexity might influence the magnitude and speed of a selected articulatory movement.

Upper lip/lower lip correlation. The same articulatory gesture from the target utterance (the bilabial closure during the second syllable of the word pompom) was used to measure the correlation between the upper lip and lower lip displacement records. This quantified the degree to which the upper lip was lowering as the lower lip was rising. A correlation of -1 would indicate that the upper and lower lip moved in exact opposition to each other (Dromey, Reese, \& Howey, 2007), as would be predicted for a bilabial opening or closing gesture. This measure was used to examine whether increasing linguistic demands would influence the coordination of lip movements. This measure differs from the oral aperture stability index used in other recent studies (MacPherson \& Smith, 2013).

Spatiotemporal Index (STI). Ten segmented and error-free displacement waveforms of the target phrase open boxes of pompoms were normalized with respect to time and amplitude for each repetition block. Amplitude was normalized by subtracting the mean and dividing by the standard deviation of each displacement. Time was normalized by Fourier analysis and resynthesis to perform a linear interpolation, as described by Smith et al. (1995). The STI was calculated by summing the displacement standard deviations from 50 equally spaced points along the normalized waveforms. This allowed the STI to measure consistency of speech articulation over multiple repetitions, where a smaller STI value reflects lower variability or greater movement stability.

Sound pressure level (SPL). The mean SPL for the target utterance open boxes of pompoms was calculated from the sound level meter output. This measure was made in order to learn whether grammatical complexity would influence vocal intensity. Previous work in our lab 
(Dromey et al., 2005) has revealed increases in vocal intensity during the performance of concurrent tasks (including linguistic challenges), and it was reasoned that the level of grammatical complexity in the present study might be associated with changes in vocal intensity.

\section{Statistical Analysis}

Univariate repeated-measures ANOVA tests were used to evaluate the statistical significance of differences in the dependent variables across the different levels of linguistic complexity. Because preliminary testing revealed no differences in the performance of men and women, the data for both were pooled for further analysis. An initial repeated-measures ANOVA included each of the five utterances, with concurrent contrast analyses comparing each complexity level against the phrase-only condition. This testing was performed to learn whether the length and complexity might interact, as was suggested by Maner et al. (2000). After interpretation of these results, a four-level repeated-measures ANOVA test, excluding the phrase-only condition, was completed to compare each linguistic complexity against the counting task, so that the syllabic length was the same for each condition. This testing was done to evaluate the effect of linguistic complexity without the influence of utterance length.

Significant within-subjects effects for linguistic complexity or between-subjects effects for age were examined with either a concurrent contrast or Tukey HSD post hoc test, respectively.

\section{Results}

Descriptive statistics for the kinematic and intensity variables for each level of sentence complexity are reported in Table 2. In the text below, where Mauchly's test showed violations of the sphericity assumption in the repeated measures ANOVA, Huynh-Feldt corrections were applied, resulting in non-integer degrees of freedom. Effect sizes are reported as partial eta squared $\left(\eta_{p}{ }^{2}\right)$. 


\section{Initial ANOVA Including the Phrase-Only Condition}

Effects of age. There was a significant between subjects effect of age on the duration of the target phrase, $F(2,57)=17.015, p<.001, \eta_{p}{ }^{2}=.374$. Post hoc Tukey HSD testing revealed that the 60 s group had a significantly longer duration than the 40 s or the 20 s, both with $p<.001$. These differences can be seen in Figure 2. There was also a modest effect of age on the correlation between upper and lower lip movements, $F(2,57)=3.202, p=.048, \eta_{p}{ }^{2}=.101$. The younger speakers had more strongly negative correlations; post hoc testing revealed this effect to be significant only between the 20 s and the 60 s $(p=.042)$. STI for the jaw differed significantly as a function of age, $F(2,56)=7.714, p=.001, \eta_{p}{ }^{2}=.216$. Post hoc testing revealed that jaw STI was lower for the 40 s than for the 20s. No significant differences were found in lower lip STI when comparing the phrase-only condition with any of the other sentences.

Effects of complexity. Utterance duration changed significantly across the conditions, $F(3.043,173.462)=27.281, p<.001, \eta_{p}{ }^{2}=.324$; concurrent contrasts revealed that the phraseonly duration was longer than for all other conditions $\left(p<.001, \eta_{p}{ }^{2}\right.$ ranged from .327 to .545$)$. The lower lip displacement for the closing gesture also differed significantly across the conditions, $F(3.892,221.856)=4.627, p=.001, \eta_{p}{ }^{2}=.075$. The phrase-only condition had a greater displacement than the baseline-counting $\left(p=.007, \eta_{p}{ }^{2}=.122\right)$ and the embedding $2(p=$ $\left..002, \eta_{p}{ }^{2}=.160\right)$ conditions. There were significant changes in lip closing velocity across the five conditions, $F(3.882,221.299)=2.800, p=.028, \eta_{p}{ }^{2}=.047$. Contrasts revealed that the velocity was greater for the phrase-only than for the baseline-counting condition $\left(p=.023, \eta_{p}{ }^{2}=.088\right)$. SPL was significantly different across the conditions, $F(3.454,193.442)=9.472, p<.001, \eta_{p}{ }^{2}=$ .145. In the ANOVA contrasts, SPL was found to be significantly higher for the phrase-only than for the baseline-counting $\left(p<.001, \eta_{p}{ }^{2}=.281\right)$ and the embedding $2\left(p=.003, \eta_{p}{ }^{2}=.150\right)$ 
conditions. The correlation between the upper and lower lips for the closing gesture changed significantly across the conditions, $F(3.746,213.541)=4.262, p=.003, \eta_{p}{ }^{2}=.070$. Contrasts revealed that the correlation was more strongly negative (suggesting greater coordination) for each of the longer sentences compared to the phrase-only condition ( $p$ ranged from .001 to .029 , $\eta_{p}{ }^{2}$ from .081 to .178). STI for the upper lip changed across the sentence conditions, $F(3.623$, $202.884)=4.319, p=.002, \eta_{p}{ }^{2}=.072$. Contrasts revealed that the upper lip variability was greater for the baseline-counting than the phrase-only condition $\left(p=.001, \eta_{p}{ }^{2}=.166\right)$.

\section{ANOVA for Sentences of Equal Length}

The phrase-only condition was excluded from subsequent analyses because it was substantially shorter than the other four sentences. In the remaining analyses, each complexity condition was compared against the baseline-counting condition to evaluate the effects of grammatical complexity without the influence of overall utterance length.

Duration. There was a group effect for age on phrase duration, $F(2,57)=16.960, p$ $<.001, \eta_{p}{ }^{2}=.373$. Post hoc testing revealed that the $60 \mathrm{~s}$ had a longer duration than the $20 \mathrm{~s}$ ( $p$ $<.001)$, as shown in Figure 2. The interaction of age with complexity was significant, $F(4.645$, $132.368)=2.405, p=.044, \eta_{p}{ }^{2}=.078$. In the 20s group, duration increased between the counting and embedding 1 conditions, whereas the 40s and 60s groups demonstrated an overall decrease in duration as complexity increased. No significant differences were found in duration across the grammatical complexity conditions.

Displacement and velocity. No differences by age were significant for displacement or velocity. Differences in lower lip displacement for the bilabial closing gesture, were significant across the levels of grammatical complexity $F(2.891,164.764)=3.611, p=.016, \eta_{p}{ }^{2}=.060$. 
Within subject contrasts revealed significantly greater displacement for the conjoining complexity compared to baseline-counting, $F(1,57)=6.847, p=.011, \eta_{p}{ }^{2}=.107$.

Peak velocity also changed significantly across the complexity conditions $F(2.87,163.571)=3.205, p=.027, \eta_{p}{ }^{2}=.053$. In the conjoining condition the velocity was higher than in the counting condition, $F(1,57)=8.534, p=.005, \eta_{p}{ }^{2}=.130$.

Upper lip/lower lip correlation. There was a significant between subjects effect for age $F(2,57)=3.308, p=.044, \eta_{p}{ }^{2}=.104$. Post hoc testing showed that the 60 s group had weaker correlations between the upper and lower lip during the bilabial closing gesture than the 20s $(p=$ .039). No significant differences were found in the upper lip/lower lip correlation across the complexity conditions and no interactions between age and complexity were significant.

STI. For the jaw STI there was a significant between subjects effect for age $F(2,56)=$ $7.411, p=.001, \eta_{p}{ }^{2}=.209$; post hoc testing revealed that the 20 s had a higher jaw STI value than the 40s $(p=.001)$.

STI of the upper lip changed significantly across the complexity conditions $F(2.665,149.252)=4.518, p=.006,=\eta_{p}{ }^{2} .075$. The conjoining condition, $F(1,56)=10.814, p=$ $.002,=\eta_{p}{ }^{2} .162$, and the embedding 2 condition, $F(1,56)=6.236, p=.015, \eta_{p}{ }^{2}=.100$, had lower STI values than the baseline-counting condition.

SPL. No interactions between age and complexity were found for intensity, and no age group differences were significant. Speech intensity differed significantly across the complexity conditions, $F(2.814,157.557)=11.604, p<.001, \eta_{p}{ }^{2}=.172$. The intensity for the baselinecounting condition was significantly lower than all of the more grammatically complex conditions ( $p$ ranged from $<.001$ to $.015, \eta_{p}{ }^{2}$ ranged from .101 to .245 ). 


\section{Discussion}

This study was designed to investigate the potential effects of age and increasing linguistic complexity on articulator movements. Several of the results suggest that speech motor control may change beyond young adulthood.

\section{Age Effects}

Utterance duration was significantly greater in the 60s group when compared to productions by the other two age groups; this is consistent with previous accounts reporting slower speech with advancing age (Harnsberger et al., 2008; Sadagopan et al., 2013). The longer utterance durations coincided with generally higher displacement and lower velocity measures, although these did not differ significantly. It could be speculated that the longer durations from the 60s group may have been necessary to complete the larger articulator movements. Benjamin (1997) noted that older adults, who experience a gradual decline in neuromotor function, may rely on slower speech rate to maintain articulatory precision. Taken together with the higher STI values for the younger speakers, it is possible that the older adults relied on a slower speech rate to maintain consistency, as well as precision, in articulator movements across repetitions of the utterance. A recent kinematic study has suggested that while older adults are capable of high peak velocities for lip and jaw movements, their fine force control appears to diminish, and they may rely on slower habitual speaking rates in order to compensate for declining articulatory control (Mefferd et al., 2014).

An unexpected finding of this study was the observation that upper lip and lower lip correlation differed across the age groups. The 20 s group had a significantly stronger negative correlations between the upper and lower lips than the 60s group. In previous studies (Dromey et al., 2005; Dromey et al., 2003), although not statistically significant, stronger upper and lower lip 
correlation was associated with slightly lower STI values, or more consistent speech movements across repetitions. The current results were not in agreement with those findings; the 20s group demonstrated the highest STI values, yet the strongest negative correlation. This suggests that while the coordination of upper and lower lip movements appeared stronger for the young adults, the consistency of their movements across repetitions was lower, potentially implicating different mechanisms to regulate coordination and consistency.

There were significant differences in speech motor stability, as reflected by jaw STI, across age groups. It was anticipated that a highly practiced and skilled motor task, such as speech production, would have fairly low levels of variability once individuals have reached adulthood. Analysis of STI results, however, indicated that the 20s group had significantly higher jaw movement variability in their speech when compared to the 40s group. Many previous studies have examined the effect of age on speech movements but have not assessed differences that occur after adulthood (Kleinow \& Smith, 2006). Green, Moore, and Reilly (2002) found that when comparing ages ranging from infancy (11-13 months) to adulthood (ages 27-35), children at the age of six produced adult-like upper lip, lower lip, and jaw movements during productions of mama, papa, and baba. These findings suggest that by the age of six, simple bilabial patterns had developed to near-adult levels. Maner et al. (2000) examined the effect of age on speech stability by comparing 5 year-old children with young adults (ages 20-25). This study found that there were still significant differences between adult and child STIs across conditions of greater linguistic complexity than just repetition of CVCV syllables. These two studies would suggest that as age increases, speech movements become more stable, possibly due to the years of experience in mastering speech motor activity. Neither of these experimental designs, however, included individuals between the ages of six and 20 and neither examined participants within a 
range of ages reaching into later adulthood. Recent findings from Sadagopan and Smith (2013), comparing the performance of younger and older adults, revealed that lip aperture variability was similar for both groups of speakers, but that the older adults made more speech errors, particularly with longer and more complex stimuli. Data from the current study suggest that the control of consistent articulatory movements continues to develop and change with age. Additional research with individuals beyond adolescence or young adulthood could provide insight into the maturation of speech motor control during phases of the lifespan that have not traditionally been viewed as periods of development.

An important consideration in discussing the differences in STI is the interpretation of this measure. On the one hand, low STI values can be interpreted as reflecting greater stability. The 40s group had the lowest STI of all the speakers when averaged across articulators and conditions. This may suggest that they showed more stable control of speech movements than the speakers in their 20s. Higher STI for the fluent speech of people who stutter (Kleinow et al., 2000) suggests that greater movement variability might be a feature of disordered speech motor control. STI was somewhat higher, averaged again across articulators and conditions, in the 60s than in the 40 s, possibly indicating decrements in motor control with advancing age. The longer durations found in the utterances produced by speakers in the 60s may have contributed to this elevated STI, since Smith et al. (1995) reported higher STI for slower speaking rates. However, in this 1995 study, the slower speech represented a departure from the speaker's habitual rate, which may not involve the same control processes as the slower habitual rate observed in the older speakers in the present work. When interpreting STI values, another perspective could be taken. Rather than viewing lower STI values as optimal, they could be viewed as evidence of reduced flexibility or plasticity, suggesting more rigid control strategies. In summary, it should 
be recognized that it is not possible to unambiguously interpret STI values. While they reflect the degree of variability across repetitions of an utterance, the underlying source and meaning of the variability can only be speculated.

\section{Length and Complexity Effects}

Due to the cognitive demands placed on the central nervous system during speech and language production, it was anticipated that increased linguistic complexity might reduce the resources allocated to producing stable speech movements. The results did not offer convincing evidence of increasing articulatory instability due solely to changes in linguistic complexity. The initial statistical analysis, which included all five complexity levels, revealed significant differences in the dependent measures across the speaking conditions. The primary differences were between the phrase-only condition and each condition of greater length and complexity. However, because fewer differences were found in subsequent testing between the complexity levels themselves (when length was held constant), the significant results suggest that length and complexity combined, rather than grammatical complexity alone, may have been responsible for most of the changes observed in this study. This had previously been speculated by Maner et al. (2000), Kleinow and Smith (2006), and MacPherson and Smith (2013). Because length appears to have confounded any linguistic complexity effect, it cannot be concluded that the differences revealed in this analysis were due to complexity alone. The initial statistical analysis confirmed that length of utterance did have an effect on speech movements, although complexity cannot be dismissed from consideration in this result, since the shorter phrase-only condition was also the simplest grammatically.

The duration of the target phrase was significantly longer in the phrase-only condition than when the same words were embedded into longer and more complex sentences. The lower 
lip displacement and velocity were also greater in this condition, as was the SPL. It could be speculated that the speakers allocated relatively more attention to speaking the phrase by itself, whereas for the longer and more complex sentences they may have put less effort into producing the target phrase because the motor planning involved many additional elements beyond the target phrase alone.

The remaining discussion examines the results of the four-level repeated-measures ANOVA, including the counting, conjoining, and embedding conditions, without the shorter phrase-only condition.

Analysis of the four complexity levels of equal length revealed significant increases in lower lip displacement and velocity for the conjoining condition. STI of the upper lip was lower for the conjoining and embedding 2 conditions. These differences were in comparison to the baseline-counting condition, which also had a lower SPL than the more complex sentences. STI of the upper lip was significantly higher, indicating less stability, for the counting condition, which was reasoned to be the least complex of the utterances, requiring the smallest linguistic demands, and having the least potential effect on articulatory stability. These findings will be considered below in the context of task naturalness.

Abbeduto (1985) examined the effect of linguistic complexity on speech production by measuring syllable duration for rapid repetitions of a simple or complex phrase. The adults in his study produced the complex sentences with longer and more variable syllable durations. Maner et al. (2000) also predicted increasing variability as complexity increased, but could not confidently attribute the results to syntactic changes due to length interference. In a study by Kleinow and Smith (2000), the authors noted no significant differences in STI of typical adults across linguistic complexity levels. The current results contrasted with each of these previous 
studies, with a simple sentence resulting in lower SPL and higher STIs than any other level of sentence complexity. It can thus be speculated that these results may not have been due solely to changes in linguistic complexity but that alternative explanations should be considered, as discussed below.

\section{STI, Speech Rate, and Naturalness}

In the current study, it was found that the counting task had higher STI, lower intensity, smaller displacement, and lower velocity than the sentences with higher complexity. There were no significant differences across conjoining or embedding complexities when compared against each other. It could be inferred that the counting condition was intrinsically different from the others, and the differences in the dependent measures cannot be confidently attributed to linguistic complexity. One possible explanation for these results may be the speed with which individuals completed the counting part of the utterance that occurred before and after the phrase that was measured. Automatic speech tasks, including rote counting, can be completed at a faster pace than linguistically demanding tasks, such as reading and producing a more complex sentence. Since the counting task alone included automatic speech, and significant differences in STI were found when comparing this condition against the others, it could be speculated that more rapid articulation when speaking the numbers may have caused a decrease in motor speech stability during the target phrase itself. However, this explanation would be inconsistent with the findings of previous studies. Smith et al. (1995) found that that a slower speaking rate resulted in higher STI, or less stable speech movements, while an increase in speech rate had no significant effect on STI. However, it should be noted that the speakers in the study by Smith and colleagues were asked to deliberately adjust their speaking rate away from their habitual speed, and that this 
may have contributed more to the STI changes than an unintended difference in rate associated with age or other factors, such as rote counting.

Another possible explanation for the higher STI and lower intensity for the counting condition is the atypical nature of the task. Each of the other complexity conditions involved a sentence structure that could be found in normal conversation, but the counting condition entailed the abnormal task of switching between automatic speech and meaningful speech, then back again. Such an unaccustomed structural switch might have had an impact on the overall cognitive demands of the task, which could contribute to the change in motor speech

performance. The demands placed on the system during the unnatural task of switching within an utterance could be a potential source of the STI and intensity differences between the counting condition and the other complexity conditions.

\section{Prosodic Variation}

The sentence context in which the target phrase open boxes of pompoms was produced may have influenced the results. As the target utterance was placed within different syntactic structures, a variety of stress, pause, and intonation patterns were naturally present. These incidental changes in prosody or speech rhythm might account for some of the differences in STI of the upper lip between the counting condition and conjoining or embedding conditions. Sentences were not equalized with respect to the number of natural pauses within them, and the target phrase was in some cases nearer the beginning and in others the end of the sentence. It could be speculated that these factors had an effect on the way in which the target utterance was spoken. Coarticulation could also have played a role in the significant differences observed across complexity levels. For instance, more complex motor movements in the words leading up 
to the target phrase might have caused more hesitation, greater emphasis, or slowed rate of the target utterance itself.

\section{Ecological Validity}

Few significant differences in articulatory stability were found across levels of linguistic complexity, suggesting that in this particular study, linguistic complexity might not have placed sufficient demands on the available resources to negatively influence articulator movement. This finding may also have been related to limitations of the experimental design. In order to use STI as a dependent measure, which required 10 repetitions of a single utterance, a structured experimental design was necessary. The repetitive nature of the stimuli, however, resulted in utterances that were not representative of typical conversational speech. The most natural form of speech production is found during everyday conversation, where linguistic formulation and speech planning occur simultaneously (Strand, 1992). An experimental design including

spontaneous conversational samples would limit the independent control of aspects of the target utterances, including length, syntax, or word choice, that were important to standardize in addressing the main research objectives of the study. The structure of the current experimental design provided a way of measuring and analyzing specific aspects of language and speech; however, no linguistic creativity was required of the participants of this study, and the task was not representative of typical language and speech behavior.

\section{Conclusion}

In summary, the findings from the present study suggest that articulatory stability changes beyond young adulthood. Although few significant results were attributed to the changes in linguistic complexity across conditions, the experimental utterances may not have been reflective of the processing demands encountered in everyday language and speech 
interactions. Future work measuring linguistic and motor interactions through a more natural task could lead to increased understanding of how the two affect each other in typical conversation.

\section{Acknowledgment}

This manuscript is based on the second author's master's thesis research. We express appreciation to the participants in the study and to the David O. McKay School of Education for

financial support. Portions of this work were presented at the annual convention of the American Speech-Language-Hearing Association in Chicago, IL, November 2013. 


\section{References}

Abbeduto, L. (1985). The effects of linguistic complexity on children's and adults' motor programming of speech. Language and Speech, 28 ( Pt 4), 361-375.

Adams, S. G., Weismer, G., \& Kent, R. D. (1993). Speaking rate and speech movement velocity profiles. Journal of Speech and Hearing Research, 36, 41-54.

Ballard, K. J., Robin, D. A., Woodworth, G., \& Zimba, L. D. (2001). Age-related changes in motor control during articulator visuomotor tracking. Journal of Speech, Language, and Hearing Research, 44, 763-777.

Barlow, S. M., Cole, K. J., \& Abbs, J. H. (1983). A new head-mounted lip-jaw movement transduction system for the study of motor speech disorders. Journal of Speech and Hearing Research, 26, 283-288.

Benjamin, B. J. (1997). Speech production of normally aging adults. Seminars in Speech and Language, 18, 135-141.

Cheung, H. \& Kemper, S. (1992). Competing complexity metrics and adults' production of complex sentences. Applied Psycholinguistics, 13, 53-76.

Dromey, C. \& Bates, E. (2005). Speech interactions with linguistic, cognitive, and visuomotor tasks. Journal of Speech, Language, and Hearing Research, 48, 295-305. 
Dromey, C. \& Benson, A. (2003). Effects of concurrent motor, linguistic or cognitive tasks on speech motor performance. Journal of Speech, Language, and Hearing Research, 46, $1234-1246$.

Dromey, C., Reese, A., \& Howey, S. (2007). Lip kinematics in spasmodic dysphonia before and after treatment with botulinum toxin. Journal of Medical Speech-Language Pathology, 15, 263-277.

Fraser, S. \& Bherer, L. (2013). Age-related decline in divided-attention: from theoretical lab research to practical real-life situations. WIREs Cognitive Science, 4, 623-640.

Givon, T. (1991). Markedness in grammar: Distributional, communicative and cognitive correlates of syntactic structure. Studies in Language, 15, 335-370.

Green, J. R., Moore, C. A., \& Reilly, K. J. (2002). The sequential development of jaw and lip control for speech. Journal of Speech, Language, and Hearing Research, 45, 66-79.

Harnsberger, J. D., Shrivastav, R., Brown, W. S., Jr., Rothman, H., \& Hollien, H. (2008). Speaking rate and fundamental frequency as speech cues to perceived age. Journal of Voice, 22, 58-69.

Illes, J., Metter, E. J., Hanson, W. R., \& Iritani, S. (1988). Language production in Parkinson's disease: acoustic and linguistic considerations. Brain and Language, 33, 146-160.

Kleinow, J. \& Smith, A. (2006). Potential interactions among linguistic, autonomic, and motor factors in speech. Developmental Psychobiology, 48, 275-287. 
Kleinow, J. \& Smith, A. (2000). Influences of length and syntactic complexity on the speech motor stability of the fluent speech of adults who stutter. Journal of Speech, Language, and Hearing Research, 43, 548-559.

MacPherson, M. K. \& Smith, A. (2013). Influences of sentence length and syntactic complexity on the speech motor control of children who stutter. Journal of Speech, Language, and Hearing Research, 56, 89-102.

Maner, K. J., Smith, A., \& Grayson, L. (2000). Influences of utterance length and complexity on speech motor performance in children and adults. Journal of Speech, Language, and Hearing Research, 43, 560-573.

Mefferd, A. S. \& Corder, E. E. (2014). Assessing articulatory speed performance as a potential factor of slowed speech in older adults. Journal of Speech, Language, and Hearing Research, 57, 347-360.

Murdoch, B. E., Cheng, H. Y., \& Goozee, J. V. (2012). Developmental changes in the variability of tongue and lip movements during speech from childhood to adulthood: an EMA study. Clinical Linguistics and Phonetics, 26, 216-231.

Panagos, J. M., Quine, M. E., \& Klich, R. J. (1979). Syntactic and phonological influences on children's articulation. Journal of Speech and Hearing Research, 22, 841-848.

Sadagopan, N. \& Smith, A. (2013). Age differences in speech motor performance on a novel speech task. Journal of Speech, Language, and Hearing Research, 56, 1552-1566. 
Sharkey, S. G. \& Folkins, J. W. (1985). Variability of lip and jaw movement in children and adults: Implications for the development of speech motor control. Journal of Speech and Hearing Research, 28, 8-15.

Smith, A., Goffman, L., Zelaznik, H. N., Ying, G., \& McGillem, C. (1995). Spatiotemporal stability and patterning of speech movement sequences. Experimental Brain Research, 104, 493-501.

Strand, E. A. (1992). The integration of speech motor control and language formulation in process models of acquisition. In R.Chapman (Ed.), Processes in Language Acquisition and Disorders (pp. 86-107). St. Louis: Mosby-Yearbook.

The Mathworks (2009). Matlab [Computer software]. Natick, MA. 


\section{Table 1}

List of stimulus items compared according to measures of length and complexity.

\begin{tabular}{lllll}
\hline $\begin{array}{l}\text { Number } \\
\text { of words }\end{array}$ & $\begin{array}{l}\text { Number } \\
\text { of syllables }\end{array}$ & $\begin{array}{l}\text { Number of } \\
\text { morphemes }\end{array}$ & $\begin{array}{l}\text { Grammatical } \\
\text { structure }\end{array}$ & $\begin{array}{l}\text { Stimulus } \\
\text { item }\end{array}$ \\
\hline 4 & 7 & 6 & Phrase only & Open boxes of pompoms \\
20 & 25 & 22 & Baseline-Counting & $\begin{array}{l}\text { One, two, three, four, five, six, seven, eight, } \\
\text { open boxes of pompoms, one, two, three, } \\
\text { four, five, six, seven, eight. }\end{array}$ \\
17 & 23 & Embedding-One & $\begin{array}{l}\text { The groups of new cheerleaders noticed the } \\
\text { open boxes of pompoms lying on the locker } \\
\text { room tables. }\end{array}$ \\
17 & 23 & Conjoining & $\begin{array}{l}\text { The cheerleaders were fighting about the } \\
\text { open boxes of pompoms and each was } \\
\text { grabbing the best ones. }\end{array}$ \\
& 25 & & Embedding-Two & $\begin{array}{l}\text { Cheerleaders sitting in the weight room } \\
\text { ignored the open boxes of pompoms that } \\
\text { were hitting the ground. }\end{array}$ \\
\hline
\end{tabular}


Table 2

Descriptive statistics for the kinematic and intensity measures in each complexity condition.

\begin{tabular}{|c|c|c|c|c|c|c|c|c|c|c|c|}
\hline \multirow[t]{2}{*}{ Condition } & \multirow[b]{2}{*}{ Age } & \multicolumn{2}{|c|}{$\underline{0-\text { Phrase Only }}$} & \multicolumn{2}{|c|}{ 1- Counting } & \multicolumn{2}{|c|}{ 2-Embedding-One } & \multicolumn{2}{|c|}{$\underline{\text { 3-Conjoining }}$} & \multicolumn{2}{|c|}{ 4- Embedding-Tw } \\
\hline & & $\bar{M}$ & $S D$ & $M$ & $S D$ & $M$ & $S D$ & $M$ & $S D$ & $M$ & $S D$ \\
\hline $\begin{array}{l}\text { Duration } \\
(\mathrm{ms})\end{array}$ & $40 \mathrm{~s}$ & 1237.2 & 141.0 & 1161.2 & 91.5 & 1154.1 & 84.2 & 1149.7 & 79.7 & 1145.7 & 74.7 \\
\hline \multirow{3}{*}{$\begin{array}{l}\text { LL displacement } \\
(\mathrm{mm})\end{array}$} & $20 \mathrm{~s}$ & 9.00 & 2.06 & 8.79 & 1.93 & 8.95 & 1.68 & 9.01 & 1.66 & 8.83 & 1.75 \\
\hline & $40 \mathrm{~s}$ & 9.44 & 2.13 & 9.08 & 1.76 & 9.28 & 2.30 & 9.29 & 1.98 & 9.08 & 2.07 \\
\hline & $60 \mathrm{~s}$ & 10.25 & 3.09 & 9.53 & 3.19 & $9 . .67$ & 3.19 & 10.07 & 3.57 & 9.35 & 2.84 \\
\hline \multirow{2}{*}{$\begin{array}{l}\text { LL velocity } \\
(\mathrm{mm} / \mathrm{s})\end{array}$} & $40 s$ & 132.72 & 28.86 & 130.15 & 26.67 & 132.47 & 31.82 & 133.39 & 31.43 & 130.24 & 28.01 \\
\hline & $60 \mathrm{~s}$ & 134.65 & 44.19 & 124.68 & 43.32 & 130.25 & 43.07 & 134.22 & 49.87 & 127.57 & 41.93 \\
\hline & $20 \mathrm{~s}$ & -0.51 & 0.28 & -0.57 & 0.24 & -0.61 & 0.25 & -0.60 & 0.25 & -0.62 & 0.24 \\
\hline \multirow{2}{*}{ Correlation } & $40 \mathrm{~s}$ & -0.34 & 0.48 & -0.37 & 0.48 & -0.39 & 0.48 & -0.36 & 0.51 & -0.41 & 0.50 \\
\hline & $60 \mathrm{~s}$ & -0.23 & 0.43 & -0.28 & 0.43 & -0.26 & 0.47 & -0.28 & 0.46 & -0.32 & 0.45 \\
\hline STI UL & $20 \mathrm{~s}$ & 11.89 & 3.47 & 12.76 & 3.96 & 13.17 & 3.92 & 11.88 & 4.02 & 11.89 & 2.05 \\
\hline STI J & $60 \mathrm{~s}$ & 12.57 & 2.92 & 13.21 & 3.40 & 12.33 & 1.86 & 11.52 & 3.78 & 11.73 & 2.72 \\
\hline \multirow[t]{3}{*}{ STI LL } & $20 \mathrm{~s}$ & 13.08 & 2.67 & 13.03 & 2.57 & 13.45 & 2.29 & 12.87 & 3.56 & 13.08 & 1.90 \\
\hline & $40 s$ & 11.32 & 2.74 & 12.09 & 3.55 & 11.17 & 2.04 & 11.06 & 2.69 & 11.51 & 2.30 \\
\hline & $60 \mathrm{~s}$ & 11.75 & 2.36 & 12.35 & 2.41 & 12.16 & 2.01 & 11.03 & 2.51 & 11.64 & 2.71 \\
\hline \multirow{3}{*}{$\begin{array}{l}\text { dB SPL } \\
\text { at } 100 \mathrm{~cm}\end{array}$} & $20 \mathrm{~s}$ & 57.20 & 3.95 & 56.06 & 3.24 & 57.26 & 2.69 & 57.15 & 2.97 & 56.81 & 3.49 \\
\hline & $40 \mathrm{~s}$ & 54.36 & 4.24 & 53.75 & 4.21 & 54.74 & 3.90 & 54.63 & 4.40 & 54.07 & 3.85 \\
\hline & $60 \mathrm{~s}$ & 57.21 & 5.92 & 55.84 & 5.39 & 56.20 & 5.47 & 56.51 & 5.84 & 55.91 & 5.24 \\
\hline
\end{tabular}

Note . Duration= segment duration; $\mathrm{LL}=$ lower lip (not decoupled from jaw); UL= upper lip; J= jaw; STI= spatiotemporal index 


\section{$\underline{\text { Figure Captions }}$}

Figure 1. Displacement (upper panel) and velocity (lower panel) of the lower lip (not decoupled

from the jaw) during one production of the target phrase. Each kinematic record was segmented starting at the velocity peak of the closing movement of $/ \mathrm{p} /$ in open (start), and ending at the closing velocity peak for $/ \mathrm{m} /$ at the end of pompoms (end). The arrows in the displacement waveform mark the closing gesture used to compute the point measures of displacement and peak velocity. $\mathrm{LL}+\mathrm{J}=$ lower lip combined with jaw movement.

Figure 2. Mean and standard deviation of the target phrase duration across the grammatical complexity conditions as a function of speaker age group.

Figure 3. Mean and standard deviation of the lower lip (not decoupled from the jaw) spatiotemporal index across the grammatical complexity conditions as a function of speaker age group. 

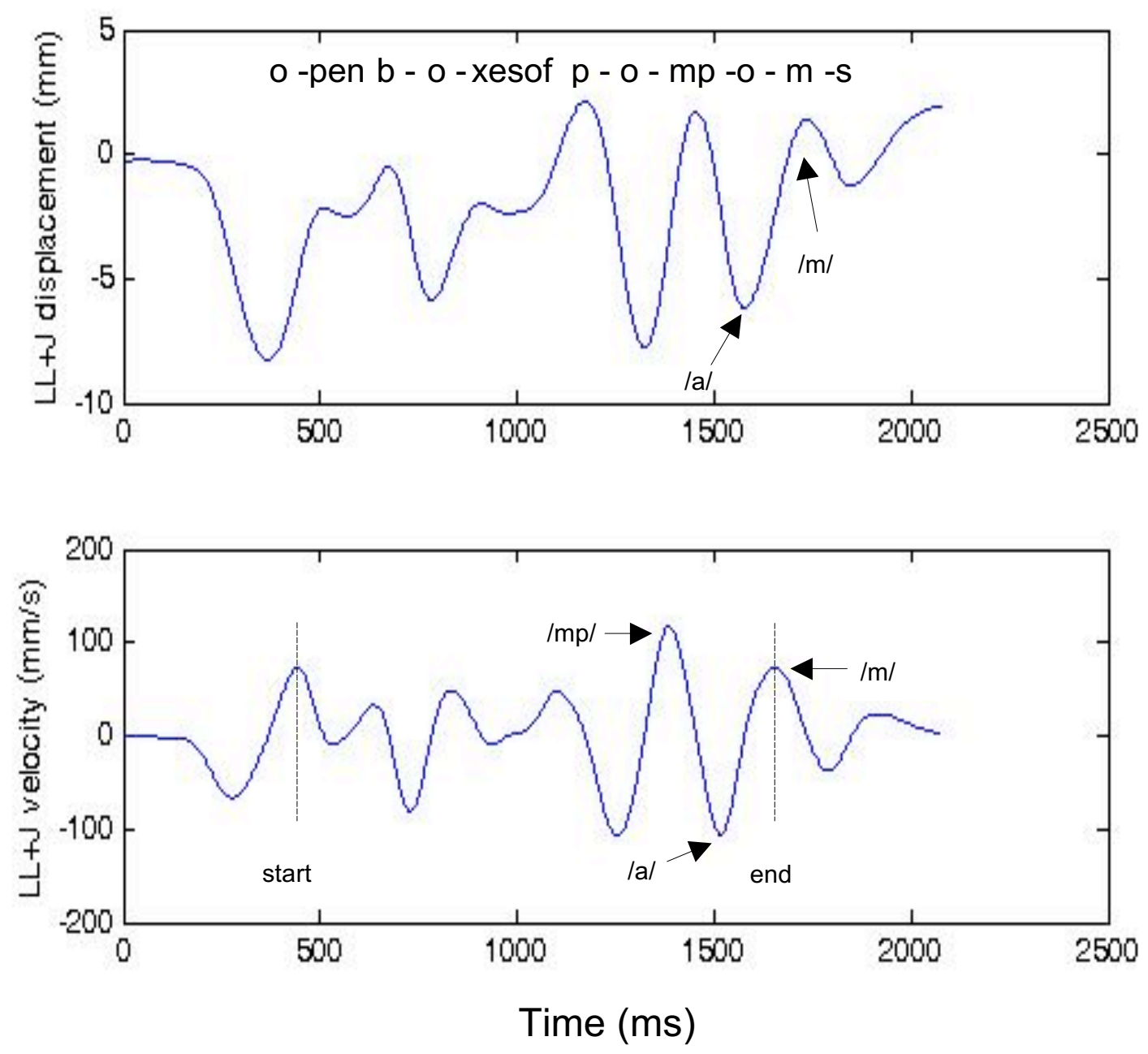


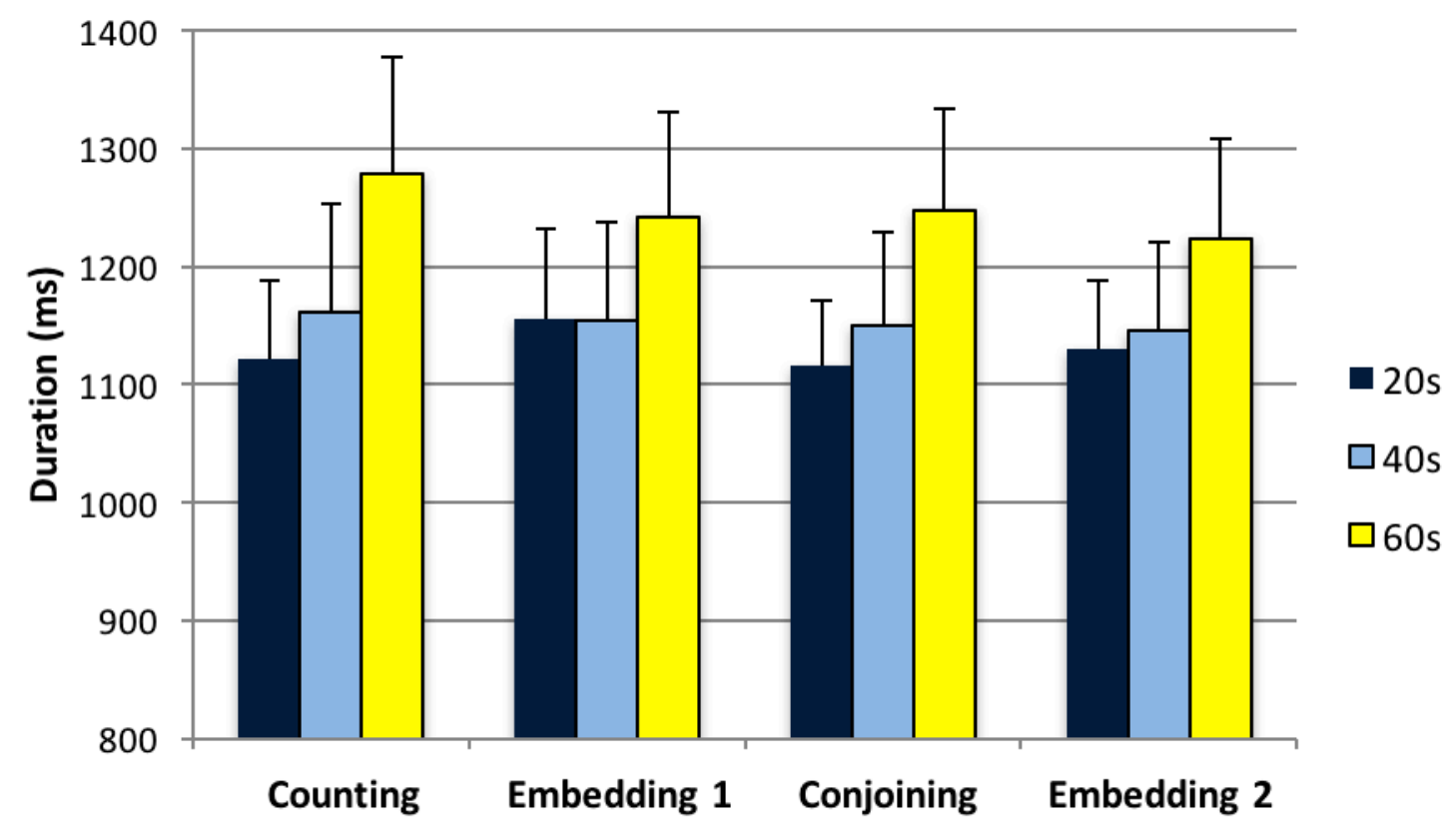




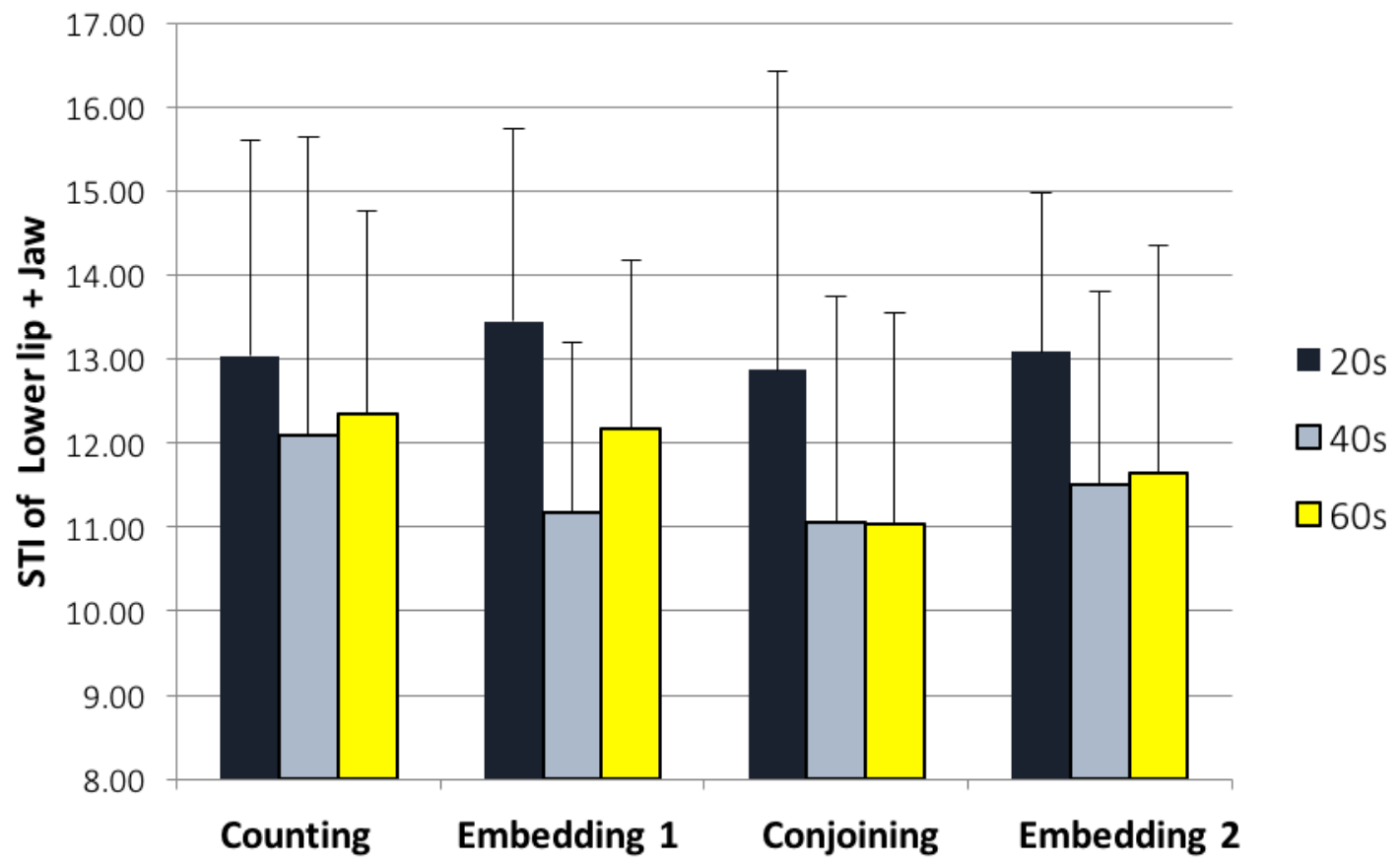

